Monatsschr Kinderheilkd 2014 • 162:722-722

DOI 10.1007/s00112-014-3150-5

Online publiziert: 26. Juni 2014

(c) Deutsche Gesellschaft für Kinder-

und Jugendmedizin
Ernährungskommission der Deutschen Gesellschaft für Kinder- und Jugendmedizin • C. Bührer · O. Genzel-Boroviczény · F. Jochum · T. Kauth · M. Kersting • B. Koletzko • W.A. Mihatsch · H. Przyrembel · T. Reinehr $\cdot$ P. Zimmer

\section{Warnung vor Verwendung unzureichend geprüfter Muttermilch}

\section{Stellungnahme der Deutschen Gesellschaft für Kinder- und Jugendmedizin}

In Deutschland wird derzeit der kommerzielle Vertrieb von humaner Milch als Spendermilch etabliert und propagiert. Die Ernährungskommission der Deutschen Gesellschaft für Kinder- und Jugendmedizin (DGKJ) warnt vor den Gefahren der Verwendung unzureichend geprüfter Spendermilch, da verbindlich geregelte, qualitätsgesicherte Kontrollen dieser Milch nicht vorgenommen werden und erhöhte Risiken durch die kommerziellen Anreize für die Verkäuferinnen bestehen.

Die beste Wahl für die Ernährung eines gesunden Säuglings ist zweifellos das Stillen [1]. Stillen bietet vielfältige Vorteile, wie z. B. eine bedarfsgerechte Nährstoffzufuhr, eine Verminderung des Risikos für das Auftreten von Infektionen beim gestillten Säugling [2-5] und positive langfristige Wirkungen wie z. B. eine Risikoreduktion für Übergewicht und Adipositas sowie eine bessere spätere kognitive Leistungsfähigkeit [2, 3, 5-8].

Mit der Verwendung von nicht umfassend kontrollierter humaner Milch zur Ernährung fremder Säuglinge sind jedoch für den Empfänger unkontrollierbare Risiken verbunden. So können über humane Milch nicht nur multiple Infektionserkrankungen, wie Humane-Immundefizienz-Virus(HIV)-Infektion oder Hepatitis, von der Spenderin auf den mit Spendermilch ernährten Säugling übertragen werden $[9,10]$, sondern auch Fremdstoffe und Wirkstoffe aus Medikamenten, die von der Spenderin eingenommen wurden [11]. Je nach Wirkstoff und Dosierung können dadurch eingreifende Schäden beim Säugling verursacht werden wie z. B. Atempausen oder lebenslang begleitende Organschäden. Außerdem ist die Zusammensetzung der hu- manen Milch von Ernährung und Ernährungszustand der Spenderin abhängig [12], sodass hierdurch weitere, schwer kontrollierbare Risiken für den durch humane Spendermilch ernährten Säugling bestehen. Darüber hinaus kann der finanzielle Anreiz für die Verkäuferin zu einem erhöhten Risiko der Kontamination durch Alkohol, Tabakbestandteile oder auch illegale Drogen mit potenziell bedrohlichen Auswirkungen führen, so wie dies bei vergüteten Blutspenden aufgetreten ist [13].

\section{Fazit für die Praxis}

Die Ernährungskommission der DGKJ rät aufgrund dieser großen Risiken - die eindeutig gegenüber möglichen Vorteilen überwiegen - dringend von der Verwendung von unzureichend geprüfter, humaner Spendermilch ab. Ausdrücklich ausgenommen von dieser Warnung sind die an wenigen Kinderkliniken in Deutschland etablierten Frauenmilchsammelstellen, die die notwendigen Sicherheitsaspekte berücksichtigen und so sichere humane Spendermilch besonders für Risikoneugeborene zur Verfügung stellen können.

\section{Korrespondenzadresse}

Prof. Dr. B. Koletzko

Ernährungskommission der Deutschen Gesellschaft für Kinder-

und Jugendmedizin e. V.

Chausseestr. 128-129, 10115 Berlin

info@dgkj.de

\section{Einhaltung ethischer Richtlinien}

Interessenkonflikt. C. Bührer, O. Genzel-Boroviczény, F. Jochum, T. Kauth, M. Kersting, B. Koletzko, W.A. Mihatsch, H. Przyrembel, T. Reinehr und P. Zimmer geben an, dass kein Interessenkonflikt besteht. Der Beitrag enthält keine Studien an Menschen oder Tieren.

\section{Literatur}

1. Koletzko B, Brönstrup A, Cremer M et al (2010) Säuglingsernährung und Ernährung der stillenden Mutter. Handlungsempfehlungen - Ein Konsensuspapier im Auftrag des bundesweiten Netzwerk Junge Familie. Monatsschr Kinderheilkd 158:679-689

2. Dutch-State-Institute-for-Nutrition-and-Health, Van Rossum CM, Büchner FL, Hoekstra J (o J) RIVM Report 350040001/2005; Quantification of health effects of breastfeeding. Review of the literature and model situation. http://www.rivm.nl/bibliotheek/rapporten/350040001.pdf

3. Agency for Healthcare Research and Quality (2007) Breastfeeding and maternal and infant health outcomes in developed countries. AHRQ Publication N $07-$ E007, S 524. http://www.breastfeedingmadesimple. com/ahrq_bf_mat_inf_health.pdf

4. Rebhan B, Kohlhuber M, Schwegler U et al (2009) Breastfeeding duration and exclusivity associated with infants' health and growth: data from a prospective cohort study in Bavaria, Germany. Acta Paediatr 98:974-980

5. Agostoni C, Braegger C, Decsi T et al (2009) Breast-feeding: a commentary by the ESPGHAN Committee on Nutrition. J Pediatr Gastroenterol Nutr 49:112-125

6. Koletzko B, Kries R von, Closa R et al (2009) Can infant feeding choices modulate later obesity risk? Am J Clin Nutr 89:1502S-1508S

7. Michaelsen KF, Lauritzen L, Mortensen EL (2009) Effects of breast-feeding on cognitive function. Adv Exp Med Biol 639:199-215

8. Steer CD, Davey Smith G, Emmett PM et al (2010) FADS2 polymorphisms modify the effect of breastfeeding on child IQ. PLoS One 5:e11570

9. Lanari M, Sogno Valin P, Natale F et al (2012) Human milk, a concrete risk for infection? J Matern Fetal Neonatal Med 25(Suppl 4):75-77

10. Lawrence RM, Lawrence RA (2004) Breast milk and infection. Clin Perinatol 31:501-528

11. Ito $S$, Lee A (2003) Drug excretion into breast milkoverview. Adv Drug Deliv Rev 55:617-627

12. Sanders TA, Reddy S (1992) The influence of a vegetarian diet on the fatty acid composition of human milk and the essential fatty acid status of the infant. J Pediatr 120:71-77

13. Offergeld R, Ritter S, Quabeck L, Hamouda O (2010) Epidemiological data on infections among blood donors in Germany 2007. Bundesgesundheitsblatt Gesundheitsforschung Gesundheitsschutz 53:11881196. DOI 10.1007/s00103-010-1152-6 